\title{
Philosophiques
}

\section{René Pellerin, Théories et pratiques de la désaliénation. Montréal, L'Hexagone, 1983, 200 p.}

\section{Éric Volant}

Volume 13, numéro 1, printemps 1986

URI : https://id.erudit.org/iderudit/203315ar

DOI : https://doi.org/10.7202/203315ar

Aller au sommaire du numéro

Éditeur(s)

Société de philosophie du Québec

ISSN

0316-2923 (imprimé)

1492-1391 (numérique)

Découvrir la revue

Citer ce compte rendu

Volant, É. (1986). Compte rendu de [René Pellerin, Théories et pratiques de la désaliénation. Montréal, L’Hexagone, 1983, 200 p.] Philosophiques, 13(1),

195-198. https://doi.org/10.7202/203315ar d'utilisation que vous pouvez consulter en ligne.

https://apropos.erudit.org/fr/usagers/politique-dutilisation/ 
RENÉ PELLERIN, Théories et pratiques de la désaliénation. Montréal, L'Hexagone, 1983, $200 \mathrm{p}$.

\author{
par Éric Volant
}

L'auteur pose la question de la teneur axiologique et des conditions d'inauguration des entreprises menées par les chercheurs ou les réformateurs. Quelle est la réalité fondatrice de leurs travaux ou de leurs interventions? Dans quelle mesure le chercheur et/ou le praticien sont-ils les auteurs, au sens propre du terme, de leurs productions? Celles-ci sont-elles le produit du milieu et trouvent-elles donc leur origine en dehors de l'individu? Des intérêts sont inévitablement à l'œuvre dans les activités scientifiques, politiques et thérapeutiques. Leurs réalisateurs sont conditionnés par des processus idéologiques, psychologiques et physiologiques. Ils sont engagés dans leurs ouvres de manière factuelle plus qu'ils ne s'y engagent d'euxmêmes à partir d'une décision qu'ils sont aptes à justifier pleinement. Or, si l'initiative du théoricien ou du libérateur échappe à une véritable prise de décision personnelle, quelle est alors son degré d'auto-implication? $\mathbf{M}$. Pellerin se propose d'interroger la logique interne qui régit l'activité du chercheur/réformateur, de mettre à jour les composantes existentielles et éthiques de leur démarche et de découvrir les facteurs d'hétéronomie qui y sont déterminants. Au fond, il s'agit d'établir le rapport qui existe entre l'agent et ses productions ainsi que le rapport entre l'agent et ses déterminismes.

L'auteur commence par définir le concept d'aliénation. L'aliénation est "l'état d'une subjectivité dont les processus vitaux (impressions, désirs, actes) ne proviennent pas d'elle-même. Cet état survient lorsqu'une réalité consciente et dynamique est privée des moyens de réaliser ce qu'elle voudrait réaliser pour assumer, obtenir ou manifester sa spécificité, comme initiateur et fondement de droit.» (p. 28). Si le premier aspect de l'aliénation est la «déprivation», le deuxième aspect est celui de la «distance»: «Aliéner, c'est défaire ou empêcher un lien avec ce qui nous appartient en propre. C'est le rejeter, l'exclure, l'abandonner à l'extérieur, renoncer à ce que l'on aliène. » (p. 30). La personne humaine est aliénée de quelque ou dans quelque chose. Dans le premier cas, elle est séparée de certaines réalités auxquelles elle voudrait ou devrait se lier ou participer. Dans le second cas, elle est liée à une réalité dont elle dépend et qui l'enchaîne. Cette situation se révèle clairement dans l'aliénation mentale. Par son discours et son comportement, l'aliéné mental est séparé ou exclu de la communauté. Par ailleurs, il est lié à cette même collectivité dont il dépend et qui l'empêche d'assumer ses décisions. L'aliénation est, en fin de compte, un processus de désappropriation par lequel l'être humain ne peut plus signer ses œuvres et peut aller jusqu'à perdre son statut de personne.

Une fois défini le concept d'aliénation, l'auteur étudie le réseau de croyances et de valeurs, les objectifs ultimes et fondamentaux, les évidences et les préférences morales des théoriciens et des thérapeutes de l'aliénation. 
Ceux-ci ne peuvent pas revendiquer pleinement pour eux-mêmes leurs investissements scientifiques, thérapeutiques ou sociaux. À l'instar de la critique de la culture marquée par la culture et à l'instar de l'interprétation de l'histoire marquée par l'histoire, l'étude et la thérapie de l'aliénation sont marquées par l'aliénation. Les chercheurs et les réformateurs s'imposent des devoirs dont ils ne savent pas si et quand ils les ont assumés comme obligation. Certaines croyances, inculquées par la culture sur la base de besoins biologiques ne sont jamais remises en question et ne semblent pas réclamer de justification rationnelle. Cette forme d'aliénation n'est pas nécessairement négative. En effet, on peut être conscient d'un acte et bien le gérer sans l'avoir inauguré. On peut être l'exécutant intelligent de ses œuvres sans en être l'initiateur.

En dehors de la perspective déterministe traditionnelle, les entreprises théoriques ou pratiques ne sont intelligibles que si l'on pose «une sorte de détermination première, un fait originaire constituant une "motivation" elle-même immotivée.» (p. 70). C'est ainsi que l'auteur introduit la notionclé de son livre. Celle-ci n'est nulle autre que l'espoir qu'il décrit comme une orientation vers des valeurs, la perception et la valorisation des possibles au-delà des faits actuels, une confiance avertie grâce à laquelle on décide de faire en mieux, en présumant que l'engagement que l'on prend ou le refus que l'on oppose soient justes et corrects. On ose croire que les résultats positifs soient possibles et souhaitables. En tant qu'événement indécidable que la conscience reçoit et à laquelle elle consent, l'espoir est une forme d'aliénation. Il est l'expérience de vouloir que quelque chose advienne sans pouvoir le produire nous-mêmes. En même temps, l'espoir constitue déjà une amorce de désaliénation en tant qu'ouverture créatrice de possibles meilleurs. La situation courante de l'humanité de l'homme, c'est l'aliénation, la désaliénation serait son achèvement, processus sans cesse à reprendre et toujours inachevé.

L'aliénation éthique positive marque toutes les décisions et interventions humaines volontaires. Elle met en évidence l'absence d'une justification finale, définitive, universelle de n'importe quelle valeur. Or, la valeur d'une valeur ne peut pas être établie sur le plan rationnel. En effet, «il faudrait savoir ce qu'est notre devoir, c'est-à-dire ce qu'il faut que nous fassions de notre propre volonté. Il faudrait ensuite ou, en même temps, décider d'assumer le devoir dont nous aurions obtenu la connaissance précise et certaine, et dont nous pourrions maîtriser les moyens de l'assumer. Il faudrait savoir pourquoi nous devrions vouloir ce que nous découvrons être notre devoir," (pp. 140-141). À la question: «Pourquoi comprendre et améliorer les situations aliénées des hommes? », l'auteur répond: "C'est parce que nous sommes en train d'espérer des résultats justes et moralement nécessaires ou, dit d'une façon plus modeste, psychologiquement ou socialement rentables ».

L'auteur critique les interventions moralisatrices et thérapeutiques ainsi que les techniques de planification du comportement (mode, propagande, éducation, formation professionnelle, etc.) dans la mesure où elles 
sont limitatrices de l'initiative personnelle. L'auteur les appelle des «technologies au service des désirs et des besoins ». Elles jouent sur les désirs les plus stables des individus, augmentent la sécurité et même la satisfaction des individus, mais elles renoncent à la reconnaissance d'autrui comme personne. Pour qu'une intervention soit personnalisante, il faut que les bénéficiaires espèrent que quelqu'un digne de crédibilité décide de reconnaître leur existence comme valeur et leur statut de personne, au-delà ou indépendamment de leurs œuvres.

En terminant, l'auteur décèle chez Marx une intuition qui confirmerait le bien-fondé de sa perception de la désaliénation. Selon Marx, la désaliénation n'est pas une appropriation individuelle personnelle, mais une réalisation de l'interdépendance matérielle des membres d'une collectivité. Dans ses rapports avec les autres au niveau de la production et du travail, l'individu reçoit son humanisation. En dépit du pouvoir moral qu'il accorde au processus historique et aux structures sociales, Marx aurait compris que la désaliénation ne peut pas être le produit de l'individu isolé. L'homme se réalise par une relation avec ses semblables, inspirée de respect et de gratification.

Dans cette étude rigoureuse et austère, l'auteur parvient à bien circonscrire le concept d'espoir. Se situant entre la croyance naïve en la liberté humaine radicale et les déterminismes scientifiques, il conçoit l'espoir comme un facteur événementiel qui advient à l'individu, lequel y consent sans l'avoir inauguré. Cet espoir motive l'individu à décider et à agir pour le mieux. L'auteur opte pour une perspective utilitariste lorsqu'il présente l'individu qui espère comme étant celui qui ose parler sur la rentabilité morale, politique ou psychologique de ses actes. Par le recours au concept d'espoir, l'auteur empêche la disparition du sujet. Car c'est celui-ci qui pense et qui agit consciemment et volontairement, même s'il n'est pas auteur initial et exclusif de ses activités. Cependant, l'espoir comme pari d'agir pour le mieux, d'où vient-il? Quelle est la source de cet événement? S'appuie-t-il sur la mémoire du passé, l'analyse du présent ou les prospectives de l'avenir? Sur l'expérience et la maturité de celui qui espère ? Or, si c'est le cas, dans ces diverses opérations de l'esprit, les interférences des déterminismes psychologiques, culturels et politiques abondent. Si ce n'est pas le cas, l'espoir s'appuie sur la foi en des promesses d'avenir, entretenues par les utopies ou les religions. Le caractère rationnel de celles-ci peut consister dans la critique du présent, mais non pas dans leurs représentations symboliques de l'avenir. Ou bien, l'espoir est une décision en fonction d'une situation de fait dont l'existence et la fin échappent à notre contrôle. C'est cette dernière option que l'auteur choisit. La dernière page de ce livre fort suggestif est sur ce point très illustrative: «... il faut admettre que décider, c'est sauter dans un train en marche, sans se rendre compte qu'on y a été poussé... L'aliénation est notre position dans ce train, et elle n'implique pas de sentiments nécessairement négatifs, dans la mesure où nous pouvons nous réjouir de faire le voyage, aussi bien que nous attrister de ne pas savoir où nous aboutirons... N'ayant pas posé nous-mêmes les rails, nous ne savons 
pas où ils mènent, et nous pouvons être assis dans un wagon qui inclut l'expérience que nous arriverons quelque part, ou dans un wagon qui inclut l'expérience encore indécidée, que nous n'allons nulle part. Les libérateurs et les intervenants prétendent conduire le train ou poser les rails, mais ce sont finalement de simples passagers.» (pp. 188-189).

Ce livre invite les chercheurs et les intervenants à découvrir avec beaucoup de modestie le lien organique qui les lie souvent à leur insu et confusément à la communauté. Ils ne naviguent pas comme seuls maîtres à bord d'un navire confondu avec l'océan. Ils participent, sans l'avoir décidé, à une force d'être ensemble, à un «vouloir-vivre ensemble» non rationnel et non logique, que Michel Maffesoli appelle la «conesthésie sociale». Ce ne sont pas eux les grands mandarins au pouvoir qui connaissent et imposent le «devoir-être» d'une société, mais ils partagent l'«espoir-être» et le «pouvoir-être» d'une communauté. Celle-ci cherche, dans le clair-obscur de son existence tragique, à donner signification et orientation à son quotidien individuel et collectif.

Département des sciences religieuses $U Q A M$ 